\title{
Flexible Slow and Fast Light in Optical Fibers
}

\author{
Luc Thévenaz, Sang-Hoon Chin, Kwang-Yong Song*, Miguel Gonzalez-Herraez** \\ EPFL, Swiss Federal Institute of Technology, STI-NAM Station 11, CH-1015 Lausanne \\ * Now with Dept. of Electronic Engineering, The University of Tokyo, Tokyo, Japan \\ ** On leave from Dept. of Electronics, University of Alcalá de Henares, Madrid 28805 Spain \\ Author e-mail address: Luc.Thevenaz@EPFL.CH
}

\begin{abstract}
A method to achieve an extremely wide and flexible control of the group velocity in an optical fiber is presented. Group velocities below $71,000 \mathrm{~km} / \mathrm{s}$ on one hand, well exceeding the speed of light in vacuum on the other hand and even negative group velocities can readily be obtained.

OCIS codes: (999.9999) Slow light; (060.4370) Nonlinear optics, fibers; (290.5900) Scattering, stimulated Brillouin
\end{abstract}

\section{Introduction}

Slow light is known to be a very attractive approach to achieve all-optical delay lines and to provide a timing tool for photonics signal processing. Successful experiments to widely control the light group velocity has been widely reported these past few years [1]. Extreme cases like negative group velocity have even been demonstrated. But all these experiments use special media like cold atomic gases or electronic transitions in crystalline solids working at well defined wavelengths.

We demonstrate here the possibility to optically control the signal velocity in an optical fibre. This is realized through the unprecedented approach of using the narrow band gain or loss generated by a nonlinear optical interaction, the stimulated Brillouin scattering [2,3]. The high flexibility of this interaction makes this active control possible in any type of fibre and at any wavelength, in particular in the low loss window of optical fibres. Using stimulated Brillouin scattering we have achieved nearly all results obtained using atomic transitions, from delays widely exceeding the optical pulse duration to superluminal propagation and even negative group velocity [4], as shown in Fig.1. This experiment can be realized on a tabletop in normal environmental conditions, so that it could be the platform for the development of a wide range of applications.

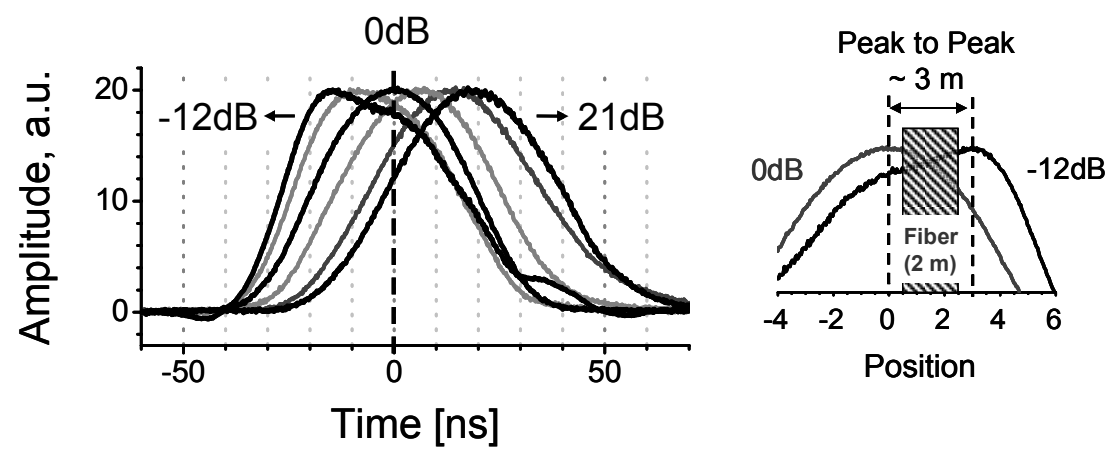

Fig. 1. Traces of the probe pulses for different Brillouin gains and losses in a 2-m test fiber, showing clear delay and advancement due to the modified group velocity. The inset compares the respective positions of the pulse propagating in normal conditions ( $0 \mathrm{~dB})$ and of the most advanced pulse $(-12 \mathrm{~dB})$, demonstrating that in this case the pulse peak exits the fiber before entering it. This situation corresponds to a negative group velocity. The fiber length is represented by the shaded area.

Actually stimulated Brillouin scattering (SBS) has proved to be an unprecedented and unmatched flexible tool for the generation of slow light regarding its spectral tailoring capability. Indeed, a large variety of gain spectral profile can be obtained by properly modulating the pump spectrum. When a monochromatic pump is used in the stimulated Brillouin interaction, the gain window appearing in the fiber transmission spectrum has a Lorentzian shape whose characteristic spectral FWHM width is around $30 \mathrm{MHz}$ in conventional single-mode fibers pumped at $1.55 \mu \mathrm{m}$. However, when the pump is modulated the gain bandwidth is given by the convolution of the pump spectrum and the Brillouin gain curve. Hence the effective Brillouin gain spectrum $g(\Delta v)$ is given by $g(\Delta v)=P(\Delta v) \otimes g_{B}(\Delta v)$ where $\otimes$ denotes convolution, $P(\Delta v)$ is the normalized pump power spectral density (i.e. its integral is unity) and $g_{B}(\Delta v)$ is the characteristic Lorentzian gain of the Brillouin amplification process.

Recently it was demonstrated that the bandwidth of SBS-based slow light can be made arbitrarily large by actively broadening the pump spectrum using random direct current modulation of the pump laser [5]. A particularly useful 
case arises when the pump spectrum can also be approximated by a Lorentzian distribution. In such conditions, the effective Brillouin gain shape remains Lorentzian, but shows a width equal to the sum of the characteristic Brillouin gain width and the pump spectral width. Hence an adequate pump modulation can be used to broaden at will the Brillouin interaction, suppressing the inherent limitation related to the narrow natural linewidth. This scheme was then replicated and improved successfully to recently ultimately reach a $10 \mathrm{GHz}$ bandwidth [6].

All these slow-light techniques suffer from the drawback of a significant amplitude change associated with the delaying effect. For instance a one-pulse width delaying gives rise to a large $30 \mathrm{~dB}$ pulse amplitude change using SBS. We demonstrate here that the high flexibility of SBS opens unprecedented achievements and offers the possibility to synthesize a gain spectral profile, so that a signal delay or advance can be realized with an ideally absolute null amplitude change. This can be obtained by the combination of gain and loss spectral profiles with identical depth but different width, resulting in a net zero gain and a differential delaying effect.

Fig. 2 left shows the obtain gain/loss spectral profile, demonstrating that a good compensation of gain and loss can be obtained. We can achieve a situation identical to ideal electromagnetically-induced transparency (EIT), that is up to full transparency.
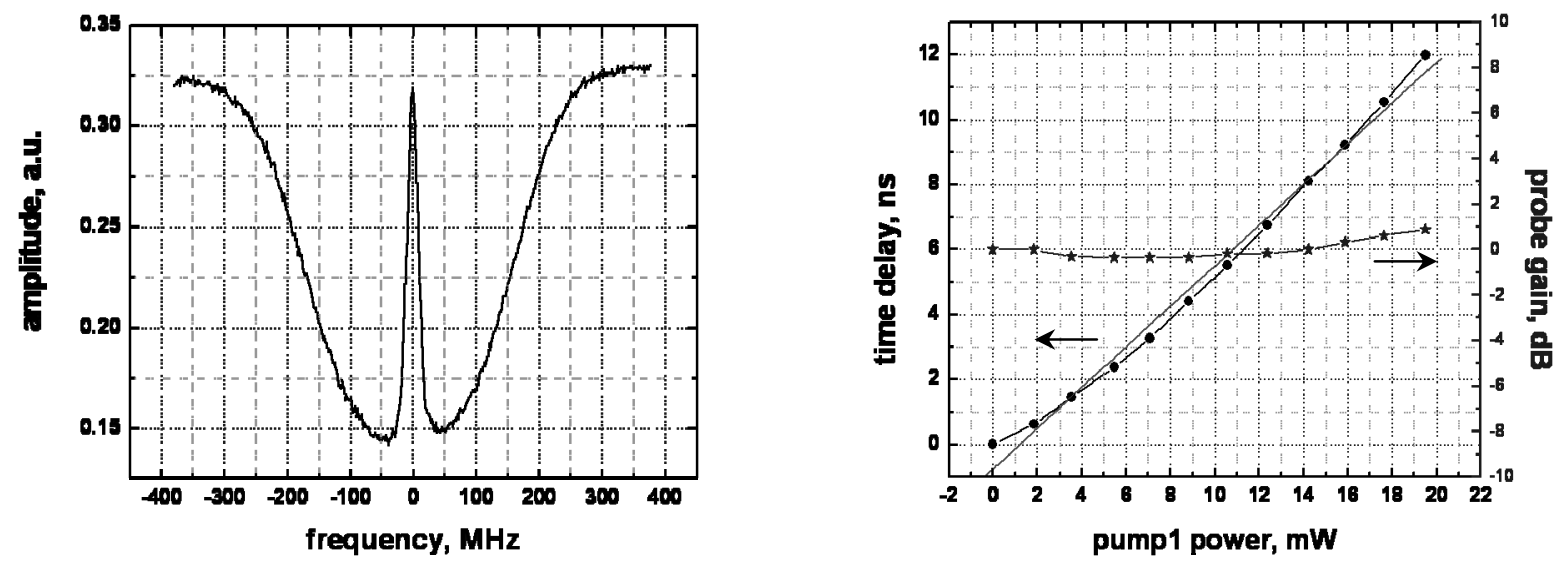

Fig.2: Left: Amplitude of a probe signal as a function of frequency after propagation in a $2 \mathrm{~km}$ fibre in which a superposed SBS gain/loss profile is generated. At the centre gain and loss fully compensate. Right: Measured delay and amplitude of a signal subject to the SBS gain spectrum shown on left, as a function of pump 1 power. The power of pump 2 is approx. 12 times higher than pump 1.

Delay and amplitude for a $1 \mathrm{MHz}$ sine modulated signal were recorded for different pump levels and are shown in Fig. 2 right. Delays comparable with the standard method [1] are obtained in a $2 \mathrm{~km}$ fiber, together with a maximum amplitude change of $\pm 1 \mathrm{~dB}$. An equivalent delay using the non-compensated standard technique would result in a $12 \mathrm{~dB}$ amplitude change. It must be pointed out that the broadband gain compensation can be produced by other types of interactions [7,8], in particular Raman, but also using doped fibers and parametric amplification. This is a significant step towards making 'slow \& fast' light not only a scientific curiosity, but a real engineering tool.

\section{References}

1 R. W. Boyd, D. J. Gauthier, “Slow' and 'Fast' Light,” Ch. 6 in Progress in Optics 43, E. Wolf, Ed. (Elsevier, Amsterdam, 2002$)$, 497-530.

2 K. Y. Song, M. Gonzalez Herráez and L. Thévenaz, “Observation of pulse delaying and advancement in optical fibers using stimulated Brillouin scattering," Opt. Express 13, 82-88 (2005).

3 Y. Okawachi, M. S. Bigelow, J. E. Sharping, Z. M. Zhu, A. Schweinsberg, D. J. Gauthier, R. W. Boyd and A. L. Gaeta, “Tunable all-optical delays via Brillouin slow light in an optical fiber," Phys. Rev. Lett. 94, 153902 (2005).

4 M. G. Herráez, K. Y. Song and L. Thévenaz, "Optically controlled slow and fast light in optical fibers using stimulated Brillouin scattering," Appl. Phys. Lett. 87, 081113 (2005).

5 M. Gonzalez Herraez, K. Y. Song, L. Thévenaz, “Arbitrary-bandwidth Brillouin slow light in optical fibers,” Opt. Express 14, 1395-1400 (2006)

6 Z. Zhu, A.M.C. Dawes, D.J. Gauthier, L. Zhang, and A.E. Willner, '12-GHz-Bandwidth SBS Slow Light in Optical Fibers,' postdeadline paper no1, in OFC/NFOEC 2006 Proceedings Series (Optical Society of America, Washington, D.C., 2006), Anaheim, CA, Mar. 5-10, 2006.

7 J. E. Sharping, Y. Okawachi, A. L. Gaeta, “Wide bandwidth slow light using a Raman fiber amplifier”, Opt. Express 13, 6092-6098 (2005).

8 D. Dahan and G. Eisenstein, "Tunable all optical delay via slow and fast light propagation in a Raman assisted fiber optical parametric amplifier: a route to all optical buffering," Opt. Express 13, 6234-6249 (2005). 